\title{
A COLLOQUIUM ON GRAPH THEORY
}

\author{
W. T. Tutte \\ (received November 12, 1959)
}

I have been asked to write a short account of the Colloquium on the theory of graphs which was held recently (October 20-22) near Budapest by the Bolyai Mathematical Society. I was invited by the Bolyai Society to participate, and owing to the generosity of the University of Toronto in the matter of my travelling expenses I was able to accept the invitation.

In addition to the many Hungarian mathematicians interested in graph theory there were a number of foreign guests, among them A.H. Stone and C.A.B. Smith of England, C. Berge of France, and H. Sachs of East Germany. I found that I was the only representative of North America. However P. Erdbs and G.A. Dirac, both of whom have spent some time with us at Toronto, were present.

The Bolyai Society paid the hotel expenses of their foreign guests, and on top of this made them a generous allowance for spending money. The Colloquium was held in a tourist hotel at Dobogókö, high in the mountains to the north of Budapest. Here we had a wide view of the Danube valley and the mountains beyond.

Summaries of the lectures given are to be published in Budapest. As the Hungarians put it, each of us made propaganda for his own region of graph theory. Smith applied graphs to the theory of games - all in Hungarian - and demonstrated by an example that his procedure could be set to music. Stone explained some of the uses that can be made in point-set topology of the graph called the "nerve" of a covering, and Berge had a graphic method for the solution of the functional equation $\mathrm{ff}(\mathrm{x})=-\mathrm{x}$. Erdys showed us some theorems to the effect that a graph with sufficiently many vertices and edges must contain subgraphs of specified structures. For myself I exhibited a new non-Hamiltonian planar cubic graph and gave an expository talk on matroids and their relation to graphs. Erdds and Dirac worked very hard translating the English lectures into Hungarian.

I have pleasant memories of talks with Hungarian graphmen outside the lecture room, mostly at meal times. Thus G. Hajos explained to me the difference between the Hajós and Hadwiger Conjectures, which are generalizations of the Four Colour Conjecture applying to non-planar graphs. Hajós is interested in ways of representing graphs. In his lecture he 
gave a theory of the representation of a graph by a set of intervals on a line. The intervals were to represent vertices, and were to overlap only for vertices joined by an edge. He drew my attention to the unsolved problem of determining what graphs can be represented by the vertices and internal diagonals of a polygon in the plane. I was glad too to meet $T$. Gallai, whose field of research is so close to my own. He explained in his lecture that he had found necessary and sufficient conditions for a graph to have a subgraph with degree 1 at specified vertices and degree 2 or 0 at each other vertex.

At the end of the Colloquium there was a meeting of Smith, Stone, Berge and myself, we being writers who used or proposed to use the English language. We wanted to agree on a uniform terminology. We decided among other things that the "degree" of a vertex ought to be called its "valency", and that a simple closed curve in a graph ought to be called a "polygon".

We foreigners were unanimous in praising the Hungarian mathematicians for the efficiency of their arrangements, and in thanking the Bolyai Society for the generosity with which we had been welcomed. 